University of South Carolina

Scholar Commons

$1-2004$

\title{
Muscular Fitness and All-Cause Mortality: Prospective Observations
}

\author{
Shannon J. FitzGerald \\ Carolyn E. Barlow \\ James B. Kampert \\ James R. Morrow Jr. \\ Allen W. Jackson
}

See next page for additional authors

Follow this and additional works at: https://scholarcommons.sc.edu/

sph_epidemiology_biostatistics_facpub

Part of the Public Health Commons

\section{Publication Info}

Published in Journal of Physical Activity and Health, Volume 1, Issue 1, 2004, pages 7-18.

FitzGerald, S. J., Barlow, C. E., Kampert, J. B., Morrow, Jr., J. R., Jackson, A. W., \& Blair, S. N. (2004). Muscular fitness and all-cause mortality: Prospective observations. Journal of Physical Activity and Health, 1(1), 7-18.

(c) Journal of Physical Activity and Health, 2004, Human Kinetics

This Article is brought to you by the Epidemiology and Biostatistics at Scholar Commons. It has been accepted for inclusion in Faculty Publications by an authorized administrator of Scholar Commons. For more information, please contact digres@mailbox.sc.edu. 


\section{Author(s)}

Shannon J. FitzGerald, Carolyn E. Barlow, James B. Kampert, James R. Morrow Jr., Allen W. Jackson, and Steven N. Blair 


\title{
O RIG INAL RESEARCH
}

Journal of Physical Activity and Health, 2004, 1, 7-18

(c) 2004 Human Kinetics Publishers, Inc.

\section{Muscular Fitness and All-Cause Mortality: Prospective 0 bservations}

\author{
Shannon J. FitzG erald, Carolyn E. Barlow, James B. Kampert, \\ James R. Morrow, Jr., Allen W. Jackson, and Steven N. Blair
}

Background: The beneficial effects of cardiorespiratory fitness on mortality are well known; however, the relation of muscular fitness, specifically muscular strength and endurance, to mortality risk has not been thoroughly examined. The purpose of the current study is to determine if a dose-response relation exists between muscular fitness and mortality after controlling for factors such as age and cardiorespiratory fitness. Methods: The study included 9105 men and women, 20-82 years of age, in the Aerobics Center Longitudinal Study who have completed at least one medical examination at the Cooper Clinic in Dallas, TX between 1981 and 1989. The exam included a muscular fitness assessment, based on 1-min sit-up and 1-repetition maximal leg and bench press scores, and a maximal treadmill test. We conducted mortality follow-up through 1996 primarily using the National Death Index, with a total follow-up of 106,046 person-years. All-cause mortality rates were examined across low, moderate, and high muscular fitness strata. Results: Mortality was confirmed in 194 of 9105 participants $(2.1 \%)$. The age- and sex-adjusted mortality rate of those in the lowest muscular fitness category was higher than that of those in the moderate fitness category ( 26.8 vs. 15.3 per 10,000 person-years, respectively). Those in the high fitness category had a mortality rate of 20.6 per 10,000 person-years. The moderate and high muscular fitness groups had relative risks of $0.64(95 \% \mathrm{CI}=0.44-0.93)$ and $0.80(95 \% \mathrm{CI}=0.49-1.31)$, adjusting for age, health status, body mass index, cigarette smoking, and cardiorespiratory fitness when compared with the low muscular fitness group. Conclusions: Mortality rates were lower for individuals with moderate/high muscular fitness compared to individuals with low muscular fitness. These findings warrant further research to confirm the apparent threshold effect between low and moderate/high muscular fitness and all-cause mortality.

Key Words: strength, prevention, prospective study, Aerobics Center Longitudinal Study

S.J. FitzGerald, C.E. Barlow, J.B. Kampert, and S.N. Blair are with the Centers for Integrated Health Research at The Cooper Institute, Dallas, TX 75230. J.R. Morrow, Jr., A.W. Jackson, and FitzGerald are with the Department of Kinesiology, Health Promotion, and Recreation at the University of North Texas, Denton, TX 76203. 
Health-related physical fitness is often described as comprising cardiorespiratory or aerobic fitness, body composition, and muscular fitness. Measures of muscular fitness include muscular strength and endurance. It is well established that cardiorespiratory fitness and aerobic activity are protective against mortality; ${ }^{1,2}$ however, the relation of muscular fitness, specifically muscular strength and endurance, to mortality risk is unclear. A few investigators have examined the relationship between grip strength and all-cause mortality and generally have found statistically significant inverse relationships after controlling for other risk factors. ${ }^{3,4,5,6}$ There has been little research on the relation of muscular endurance to mortality, although Katzmarzyk et al. ${ }^{7}$ recently suggested an inverse association between the number of sit-ups in $1 \mathrm{~min}$ and mortality in the Canada Fitness Survey.

There may be many health benefits directly and indirectly associated with muscular fitness, and these could lead to a relationship between muscular fitness and mortality. Although the evidence is equivocal, literature reviews suggest that those who perform better on strength tests tend to be healthier ${ }^{8,9}$ and have better functional capacity ${ }^{10,11}$ than those who do not perform as well. High levels of muscular strength may indirectly improve health profiles through beneficial effects on body composition and aerobic performance. ${ }^{12}$ Muscular strength and endurance training programs have minor effects on maximal aerobic power, but they may lead to improvements in submaximal exercise performance, ${ }^{12}$ thus reducing the risk for mortality indirectly by lowering the risk for functional decline and disability. ${ }^{13,14}$

The aim of the current study was to determine the relation between muscular fitness and mortality after controlling for factors such as age, health status, body mass index, cigarette smoking, and cardiorespiratory fitness. Muscular fitness, represented by a muscular strength and endurance index, included measures of upper and lower body strength and abdominal muscular endurance. We examined the dose-response relation of low, moderate, and high levels of muscular fitness to allcause mortality. We hypothesized that those in the moderate and high muscular fitness categories would have lower rates of mortality compared with those in the lowest muscular fitness category.

\section{Methods}

\section{Study Population and Design}

Data for the current study are from the Aerobics Center Longitudinal Study (ACLS). The aim of the ACLS is to examine prospectively the association of physical activity and physical fitness to health in men and women. Individuals who have completed at least one medical examination at the Cooper Clinic in Dallas, TX are participants in the ACLS. To be eligible for the current study, participants also had to have completed a muscular fitness assessment, which was offered from 19811989, as part of their medical examination. They also completed a maximal treadmill test that followed a modified Balke protocol ${ }^{15}$ and achieved at least $85 \%$ of their age-predicted maximal heart rate on the test. Annually, the Institutional Review Boards of The Cooper Institute and the University of North Texas reviewed and approved the study protocol. Prior to the examination, the participants fasted for 12 hours, were asked not to smoke on the day of the examination, and granted written informed consent for the examination and follow-up. The evaluation included a physical exam, anthropometrics, blood chemistry tests, blood pressure, a 
maximal treadmill exercise test to assess cardiorespiratory fitness, and a questionnaire of personal and family medical history, demographic characteristics, and health habits.

Following the standard examination, participants could volunteer to complete a muscular fitness assessment. No standardized contraindications precluded an individual from participating. They were allowed to volunteer as long as their examining physician did not feel that participating in the assessment would worsen an existing medical condition or place them at risk. ${ }^{16}$

\section{Clinical Examination}

Various tests were conducted as part of the muscular fitness assessment. Components of the assessment included in the current study were 1-repetition maximal bench and leg press lifts and a 1-min bent leg sit-up test. Briefly, the sit-up test consisted of the participants performing as many correct bent leg sit-ups as possible in 1 min with their hands behind their head and their feet flat on the floor, held by a technician. The 1-repetition maximal bench and leg press tests were all performed on Universal weight machines (Universal Equipment, Cedar Rapids, IA). The initial weight for the bench press was $70 \%$ of the participant's body weight. Weight was added in 2.3- to 4.5-kg increments until maximal effort was performed, usually in five trials or less. This was also done for the maximal leg press test; however, the starting weight for the participant was $100 \%$ of their body weight. These assessment protocols have previously been described more thoroughly. ${ }^{16,17}$

Maximal treadmill tests were conducted to assess cardiorespiratory fitness. Maximal oxygen uptake $\left(\mathrm{VO}_{2 \max }\right)$ is the best indicator of cardiorespiratory fitness. ${ }^{18}$ Since $\mathrm{VO}_{2 \max }$ is highly correlated ( $r=0.92$ in men and $r=0.94$ in women) with the duration of our maximal treadmill exercise test, ${ }^{19,20}$ time from the treadmill test was used to estimate cardiorespiratory fitness in the current study. The specific treadmill protocol was as follows: The treadmill speed was originally set at $88 \mathrm{~m} / \mathrm{min}$ and remained that speed for the first $25 \mathrm{~min}$ of the test. Initially, the grade was set at $0 \%$ for the first minute, then increased to $2 \%$ for the second minute, and subsequently increased $1 \%$ each minute until $25 \mathrm{~min}$ into the test. At this point, the grade stayed constant and the speed increased $5.4 \mathrm{~m} / \mathrm{min}$ each minute until the test was stopped. All participants were encouraged to provide a maximal effort performance.

Cooper Clinic laboratory technicians analyzed blood chemistry levels using automated techniques. The lab participates in and meets quality control standards of the Centers for Disease Control and Prevention Lipid Standardization Program. Blood pressure was ascertained by the auscultatory method utilizing standard procedures using a mercury sphygmomanometer with the patient in the sitting position. A standard physician's balance beam scale and stadiometer were used to measure weight and height. ${ }^{21}$ From this measurement, we estimated obesity based on body mass index (BMI) [weight $(\mathrm{kg}) /$ height $\left.\left(\mathrm{m}^{2}\right)\right]$.

\section{M ortality Ascertainment}

We conducted mortality follow-up through 1996 primarily using the National Death Index. The National Death Index has a sensitivity of $96 \%$ and a specificity of $100 \%$ for determining deaths in the general population. ${ }^{22}$ Once we identified possible decedents, departments of vital statistics in the appropriate states were contacted, 
and official copies of death certificates were requested. We compared information on the death certificates with clinical records to confirm that the death certificate matched the individual. A nosologist coded the underlying and potentially 4 other contributing causes of death according to the International Classification of Diseases (9th ed., revised; ICD-9).

\section{Statistical Analysis}

We created muscular fitness categories from a muscular fitness scoring index that was based on results from 1-repetition maximal bench and leg press tests and from a 1-min sit-up test. Tertiles were calculated for each test separately for men and women and were assigned a 0 to 2 score $(0=$ the lowest tertile, $2=$ the highest tertile). Specific cutpoints for men and women for each test according to the score are shown in Table 1. The individual scores were then summed to form the muscular fitness index ( 0 to 6 score). We investigated the cumulative incidence of allcause mortality across these scores and determined that the highest incidence of mortality was in those who scored a $0(n=54)$ on the muscular fitness index, and the lowest incidence was in those who scored a $6(n=8$, data not shown). Based on these observations, to have comparable numbers of deaths in each group, we defined muscular fitness categories as follows: low $=0$ on the muscular fitness index, moderate $=1$ to 3 , and high $=4$ to 6 . We compared descriptive characteristics of men and women across muscular fitness categories. We also conducted Pearson correlation analyses to examine the strength of the associations among ungrouped components of the muscular fitness assessment.

We calculated age- and sex-adjusted all-cause mortality rates per 10,000 person-years of observation across low, moderate, and high muscular fitness categories. The direct method was used for adjustment with the entire sample as the standard.

Table 1 Cutpoints for Muscular Fitness Index Tertiles in Women and Men, Aerobics Center Longitudinal Study

$\begin{array}{lll}\text { Bench Press, maximal kg lifted } & & \\ 0 & \leq 61.2 & \leq 27.2 \\ 1 & 61.3-74.8 & 27.3-36.3 \\ 2 & 74.9+ & 36.4+ \\ \text { Leg press, maximal kg lifted } & & \\ 0 & \leq 120.2 & \leq 63.5 \\ 1 & 120.3-140.6 & 63.6-81.6 \\ 2 & 140.7+ & 81.7+ \\ \text { Sit-ups, number in 1 min } & & 0-20 \\ 0 & 0-28 & 21-28 \\ 1 & 29-36 & 29+ \\ 2 & 37+ & \end{array}$


We used Cox proportional hazards regression to calculate multivariable adjusted relative risks (RR) ${ }^{23}$ for all-cause mortality. The log cumulative hazard plots against follow-up time were parallel across groups. These models included age, sex, smoking status, health status, cardiorespiratory fitness level, BMI, resting systolic blood pressure, serum cholesterol, and year of examination. We considered individuals apparently healthy if, at the time of their exam, they had normal resting and exercise electrocardiograms and did not report a history of myocardial infarction, high blood pressure, stroke, diabetes, or cancer. The $95 \%$ confidence intervals (CI) around all RRs were also calculated. We conducted all data analyses using SAS 8.2 statistical software (SAS Institute, Inc., Cary, North Carolina).

\section{Results}

Complete data were available on 9105 men and women, 20-82 years of age. Characteristics of the study population by muscular fitness category are shown in Table 2. More than $80 \%$ of the participants were men. Age, total cholesterol, and triglycerides were lower in men and women in the high muscular fitness category than those in the low or moderate muscular fitness category. Those in the high muscular fitness group were also less likely to be current smokers or unhealthy and had better physical activity/cardiovascular fitness profiles compared to those in the low or moderate categories. Average follow-up time for each group ranged from 11.6 to 11.7 personyears for a total follow-up time of 106,046 person-years. Approximately $4 \%$ of the total follow-up time was accounted for individuals 60 years old and older.

Correlations among the three components of the muscular fitness indexsit-up, leg, and bench press scores - are shown in Table 3. Bench and leg press scores were the most highly correlated, $r=0.69$ in women and $r=0.62$ for men, $p<.001$ for both. In men and women, sit-up score and bench press score were moderately correlated ( $r=0.48$ to 0.41 , respectively, $p<.001$ for both), and sit-up score and leg press score were weakly correlated $(r=0.22$ to 0.32 , respectively, $p<.001$ for both).

Mortality was confirmed in 194 participants (2.1\%). Characteristics of the decedents are shown in Table 4 . Over $90 \%$ of the decedents were men, and $49.5 \%$ were less than 50 years old. All of the deaths occurred between 1 and 14 years of follow-up; $43.8 \%$ occurred between 6 and 10 years. Cancer was the cause of most deaths $(45.9 \%)$, followed by cardiovascular disease $(25.2 \%)$. There were 56 deaths in the low, 84 in the moderate, and 54 in the high muscular fitness groups, respectively. Age- and sex-adjusted all-cause mortality rates per 10,000 person-years by muscular fitness category are shown in Figure 1. The rate of those in the lowest muscular fitness category was higher than that of those in the moderate fitness category (26.8 vs. 15.3 per 10,000 person-years, respectively). Those in the high fitness category had a mortality rate of 20.6 per 10,000 person-years.

Proportional hazards analyses demonstrated that those in the moderate and high muscular fitness groups had a RR of $0.56(95 \% \mathrm{CI}=0.40-0.80)$ and 0.65 (95\% CI $=0.42-0.99)$, respectively, compared with the low muscular fitness group, after adjustment for age and sex (Table 5). Test for linear trend for the categorical muscular fitness variable reached borderline significance $(p=.06)$. Test for linear contrast comparing the combined effects of the moderate and high muscular fitness groups with the low fitness group on mortality risk was statistically significant $(p<.01)$. 
Table 2 Descriptive Characteristics of Men and Women According to Muscular Fitness Category

\begin{tabular}{|c|c|c|c|c|}
\hline Variable & $\begin{array}{c}\text { Low } \\
(N=1069)\end{array}$ & $\begin{array}{l}\text { Moderate } \\
(N=4280)\end{array}$ & $\begin{array}{c}\text { High } \\
(N=3756)\end{array}$ & $p$ value \\
\hline $\operatorname{Men}(\%)$ & 87.3 & 84.6 & 81.2 & $<.001$ \\
\hline Age $(y)$ & $50.3 \pm 9.1$ & $43.6 \pm 8.8$ & $37.4 \pm 7.7$ & $<.001$ \\
\hline Total cholesterol $(\mathrm{mg} / \mathrm{dl})^{\dagger}$ & $219.5 \pm 44.4$ & $213.2 \pm 66.1$ & $202.3 \pm 40.1$ & $<.001$ \\
\hline $\mathrm{HDL}(\mathrm{mg} / \mathrm{dl})^{\dagger}$ & $48.9 \pm 13.6$ & $48.6 \pm 17.6$ & $48.0 \pm 13.1$ & .36 \\
\hline Triglycerides $(\mathrm{mg} / \mathrm{dl})^{\dagger}$ & $105.5(75,152)$ & $100(70,148)$ & $90.5(65,136)$ & $<.001$ \\
\hline Glucose $(\mathrm{mg} / \mathrm{dl})^{\dagger}$ & $101.3 \pm 16.8$ & $99.8 \pm 14.0$ & $97.9 \pm 10.6$ & $<.001$ \\
\hline $\begin{array}{l}\text { Resting systolic blood } \\
\text { pressure }(\mathrm{mm} \mathrm{Hg})\end{array}$ & $118.9 \pm 15.1$ & $116.6 \pm 13.0$ & $117.1 \pm 12.4$ & .001 \\
\hline $\begin{array}{l}\text { Resting diastolic blood } \\
\text { pressure }(\mathrm{mm} \mathrm{Hg})\end{array}$ & $79.8 \pm 9.7$ & $78.4 \pm 9.3$ & $77.9 \pm 9.1$ & $<.001$ \\
\hline $\mathrm{BMI}\left(\mathrm{kg} / \mathrm{m}^{2}\right)$ & $24.3 \pm 2.9$ & $25.0 \pm 3.5$ & $25.9 \pm 4.0$ & $<.001$ \\
\hline Unhealthy $(\%)^{\ddagger}$ & 26.3 & 18.4 & 12.4 & $<.001$ \\
\hline Current smoker $(\%)$ & 16.9 & 14.7 & 12.8 & .001 \\
\hline $\begin{array}{l}\text { Cardiorespiratory fitness } \\
\text { (maximal METs on } \\
\text { treadmill test) }\end{array}$ & $16.1 \pm 4.5$ & $18.4 \pm 4.9$ & $20.6 \pm 4.9$ & $<.001$ \\
\hline Unfit $(\%)^{\S}$ & 10.7 & 8.9 & 6.7 & .05 \\
\hline Sedentary $(\%)^{\$}$ & 28.6 & 22.1 & 16.2 & $<.001$ \\
\hline
\end{tabular}

Note. Mean $\pm S D$ or median $\left(25^{\text {th }}, 75^{\text {th }}\right.$ percentile $)$ unless indicated. $†$ To convert cholesterol and HDL values to $\mathrm{mmol} / \mathrm{L}$, multiply by 0.0259 . To convert triglycerides to $\mathrm{mmol} / \mathrm{L}$, multiply by 0.0113 . To convert glucose to $\mathrm{mmol} / \mathrm{L}$, multiply by 0.0555 . $\$$ Abnormal or equivocal resting or exercise electrocardiograms or a history of myocardial infarction, high blood pressure, stroke, diabetes, or cancer. $§$ Those in the lowest $20 \%$ age- and sex-specific cardiorespiratory fitness category. $\$$ No regular physical activity reported 3 months before the examination.

Table 3 Correlations Among Muscular Fitness Measures for Men and Women

\begin{tabular}{lcccc}
\hline & \multicolumn{2}{c}{ Men $(N=7605)$} & \multicolumn{2}{c}{ Women $(N=1500)$} \\
\cline { 2 - 5 } Measures & Leg press $^{\dagger}$ & Bench press $^{\ddagger}$ & Leg press & Bench press \\
\hline Sit-ups $^{\S}$ & 0.22 & 0.48 & 0.32 & 0.41 \\
Leg press & & 0.62 & & 0.69 \\
\hline
\end{tabular}

Note. All correlations $p<.001$. $†$ Maximal 1-repetition leg press. $\$$ Maximal 1-repetition bench press. §Number of sit-ups in $1 \mathrm{~min}$. 
Table 4 Characteristics of 194 Decedents

\begin{tabular}{lr}
\hline Variable & $\%$ \\
& \\
Men & 91.2 \\
$\geq 50$ years old & 50.5 \\
Follow-up time & \\
1-5 years & 26.8 \\
6-10 years & 43.8 \\
11-14 years & 29.4 \\
Cause of death & \\
Infectious disease & 2.6 \\
Cancer & 45.9 \\
Nutrition or metabolic disease & 2.1 \\
Nervous system & 3.6 \\
Cardiovascular disease & 25.2 \\
Digestive disease & 2.0 \\
Genitourinary disease & 0.5 \\
Injury or poisoning & 1.6 \\
Other & 16.0 \\
Unknown & 0.5 \\
&
\end{tabular}

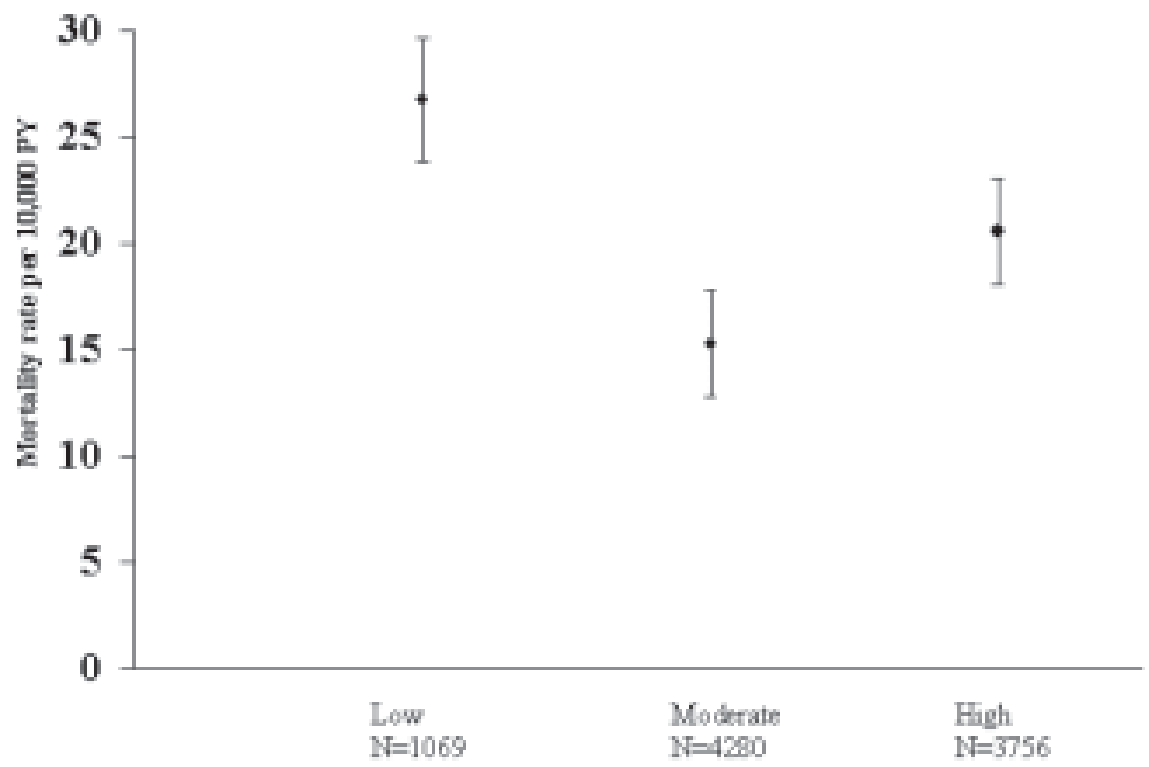

Figure 1 - Mortality rates per $\mathbf{1 0 , 0 0 0}$ person-years by muscular fitness category. 
There were no appreciable differences in results when baseline smoking and health status were added to the model (Table 5). The moderate and high muscular fitness groups had an adjusted RR of $0.58(95 \% \mathrm{CI}=0.41-0.82)$ and $0.69(95 \% \mathrm{CI}$ $=0.45-1.06$ ) when compared with the low muscular fitness group. Linear contrast comparing the mortality risk between the moderate and high muscular fitness groups and the low fitness group was statistically significant $(p=.01)$. When cardiorespiratory fitness level, BMI, cholesterol, resting systolic blood pressure, and baseline examination visit year were added to the model, the strength of the relationships was slightly diminished (Table 5). Linear contrast for this model comparing mortality risks of the moderate and high muscular fitness groups with the low fitness group was borderline significant $(p=.09)$. These analyses were repeated, excluding all individuals who died within 3 years of their clinic exam $(n=18)$. The results were not substantially changed with these exclusions (data not shown).

\section{Table 5 Relative Risks of All-Cause Mortality Across Muscular Fitness Categories}

\begin{tabular}{lll} 
Model/category & RR & 95\% CI \\
\hline $\begin{array}{ll}\text { Model } 1^{\dagger} \\
\quad \text { Low muscular fitness }\end{array}$ & \\
$\quad$ Moderate muscular fitness & 1.0 & $(0.40-0.80)$ \\
$\quad$ High muscular fitness & 0.56 & $(0.42-0.99)$ \\
Model $2^{\ddagger}$ & 0.65 & \\
$\quad$ Low muscular fitness & 1.0 & $(0.41-0.82)$ \\
$\quad$ Moderate muscular fitness & 0.58 & $(0.45-1.06)$ \\
$\quad$ High muscular fitness & 0.69 & \\
Model $3^{\S}$ & & \\
$\quad$ Low muscular fitness & 1.0 & $(0.44-0.93)$ \\
$\quad$ Moderate muscular fitness & 0.64 & $(0.49-1.31)$ \\
$\quad$ High muscular fitness & 0.80 &
\end{tabular}

Note. Fifty-six deaths in the low, 84 in the moderate, and 54 in the high muscular fitness groups. $\uparrow$ Adjusted for age and sex; $p$ for trend $=.06$. $\$$ Adjusted for age, sex, health status, and current smoking status. §Adjusted for age, BMI, sex, cardiorespiratory fitness, health status, total cholesterol, resting systolic blood pressure, smoking status, and baseline examination year.

\section{Discussion}

Mortality was confirmed in 194 of 9105 men and women over 106,046 personyears of follow-up. Mortality rates across low, moderate, and high muscular fitness categories, based on 1-min sit-up and 1-repetition maximal leg and bench press scores, suggest there is a threshold effect, rather than a dose-response relation, between low muscular fitness and moderate/high muscular fitness and allcause mortality. Better muscular fitness was associated with lower age- and sex-adjusted all-cause mortality rates. 
When examining the relation between muscular fitness and all-cause mortality using multivariate modeling, the moderate and high muscular fitness groups had, respectively, a $44 \%$ and $35 \%$ reduction in risk compared with the low muscular fitness group, after adjusting for age and sex. Further adjustment for health and current smoking status had minimal effects on the strength of the relationship, with the risk reduction being $42 \%$ and $31 \%$ for the moderate and high muscular fitness groups, respectively. Additional adjustment for cardiorespiratory fitness, body mass index, cholesterol, resting systolic blood pressure, and baseline examination year slightly diminished the level of risk.

Our results support previously published literature regarding the relation between muscular strength, a component of muscular fitness, and all-cause mortality. Prospective studies have reported that survivors had higher strength at baseline compared to those who died. ${ }^{24,25}$ Additional studies reported that the mortality risks in those with low grip strength ranged from $20 \%$ higher to over twice that of the high strength group..$^{3,4,6,26}$ Metter and colleagues reported that lower grip strength at baseline as well as declining grip strength over time were related to increased mortality. ${ }^{5}$ Rantanen et al. ${ }^{6}$ examined these relationships across BMI strata and found that the increased mortality risk for those with low grip strength was seen in those who were low weight $(\mathrm{BMI}<20)$, normal weight, and overweight/obese $(\mathrm{BMI} \geq 25)$.

There have not been many studies examining the relationship between allcause mortality and muscular endurance, another component of muscular fitness. Investigators from the Canadian Fitness Survey found that men and women in the lowest quartile for sit-up score had a significantly higher risk for all-cause mortality compared with those in the highest quartile. ${ }^{7}$ Fujita et al., however, did not find a relationship between sit-up score and mortality. ${ }^{4}$

Most of the above-mentioned studies were performed in elderly cohorts (e.g., $\geq 70$ years old) and did not include a measure of cardiorespiratory fitness. ${ }^{3,24-26}$ These studies typically investigated the relationship between one component of muscular fitness, usually strength, and mortality. ${ }^{3-6,24-26}$ For the most part, these studies focused on strength assessed in one muscle group (e.g., grip strength). A thorough strength assessment should include testing several major muscle groups, including upper body, trunk, and lower body. ${ }^{2}$ Our study group was unique in that the participants ranged in age from 20 to 82 years old and also had a measure of cardiorespiratory fitness based on a maximal treadmill test. Since moderate and high levels of muscular fitness could represent an active lifestyle, the ability to adjust for cardiorespiratory fitness allowed us to focus specifically on the relation of muscular fitness to mortality. In addition, our muscular fitness index was based on both muscular strength and endurance tests, which involved the major muscle groups of the upper and lower body and the trunk. The study results provide evidence that participants with moderate/high muscular fitness had lower mortality risk than low fit individuals.

Those who demonstrate high levels of strength tend to be healthier ${ }^{8,9}$ and have better functional capacity ${ }^{10,11}$ than those with lower levels of strength. Therefore, our results could be explained, in part, by the relationships between various components of health and muscular strength. For example, it has been suggested that low levels of muscular strength and/or endurance ${ }^{17,27}$ were predictive of poor physical function, which in turn has been found to be related to an increased mortality risk. ${ }^{13,14}$ Resistance training programs designed to improve muscular fitness have been found to improve functional mobility in the elderly; ${ }^{28,29}$ therefore, 
moderate and high levels of muscular fitness may reduce mortality risk through improvements in health indices such as functional capacity. Additional beneficial effects of resistance training programs include positive changes in bone mineral density, body composition, and glucose metabolism. ${ }^{12,30}$

Strengths of the current study include the availability of an objective multicomponent assessment of muscular fitness and cardiorespiratory fitness on a large sample of men and women. In addition, the thorough medical examination participants received at baseline allows us to control for additional risk factors such as health status, systolic blood pressure, and cholesterol in multivariate models.

Because of the demographic structure of the population, predominantly white and middle to upper socioeconomic status, the results of this study may not be applicable to those in the general population of the United States. Earlier we compared values of blood pressure, cholesterol, weight, and cardiorespiratory fitness from subjects in the ACLS to participants in two other large epidemiological studies that were conducted in representative populations in North America. ${ }^{31}$ The values for these variables did not vary substantially across studies for age- and sex-specific groups. The homogeneity of our sample on education, occupation, and income reduces the potential for confounding by these characteristics.

Other limitations include the inability to analyze data for men and women separately. This limitation was due to the low all-cause mortality rate in women resulting in a lack of statistical power. Analyses were stratified by sex, and the same trends between muscular fitness category and all-cause mortality were found in men and women separately (data not shown). These relationships did not reach statistical significance in women $(p>.05)$. It is also possible that those individuals who performed more poorly on the muscular fitness test had health conditions not detected at the examination, making them at higher risk for mortality. However, all participants received a thorough medical examination at baseline and were healthy enough to achieve at least $85 \%$ of their age-predicted maximal heart rate on a treadmill test. To further address this issue, we controlled for existing health conditions in multivariate analyses and repeated analyses, excluding those who died within 3 years of their exam. These adjustments did not alter the results substantially. Finally, we examined muscular fitness at one point in time. The muscular assessment was only offered from 1981 and 1989, and less than $8 \%$ of the sample had more than one visit (data not shown). Given the lack of data for repeat muscular fitness assessments, we were unable to examine changes or maintenance of muscular fitness over time. If changes in muscular fitness did occur, the strengths of the associations reported potentially would be affected.

To our knowledge this is the first study to report a threshold effect between low and moderate/high muscular fitness, based on muscular fitness screening, and lower all-cause mortality. Future studies should examine the relation between muscular fitness and the development of diseases such as coronary heart disease and type 2 diabetes, as well as the effects of long-term maintenance and improvements of muscular fitness on morbidity and mortality. Reports from organizations such as the Centers for Disease Control and Prevention and the American College of Sports Medicine ${ }^{1}$ and the Surgeon General ${ }^{2}$ suggest that physical activity regimens designed to improve health should include a resistance training component. Potentially, resistance training programs designed to improve total muscular fitness should be recommended not only to improve overall health and functional status, but perhaps to reduce all-cause mortality as well. 


\section{References}

1. Pate RR, Pratt M, Blair SN, et al. Physical activity and public health: a recommendation from the Centers for Disease Control and Prevention and the American College of Sports Medicine. JAMA. 1995;273:402-407.

2. U.S. Department of Health and Human Services. Physical Activity and Health: A Report of the Surgeon General. Atlanta, GA: U.S. Department of Health and Human Services, Centers for Disease Control and Prevention, National Center for Chronic Disease Prevention and Health Promotion; 1996.

3. Anstey KJ, Luszcz MA, Giles LC, Andrews GR. Demographic, health, cognitive, and sensory variables as predictors of mortality in very old adults. Psychol Aging. 2001;16:311.

4. Fujita Y, Nakamura Y, Hiraoka J, et al. Physical-strength tests and mortality among visitors to health-promotion centers in Japan. J Clin Epidemiol. 1995;48:1349-1359.

5. Metter EJ, Talbot LA, Schrager M, Conwit R. Skeletal muscle strength as a predictor of all-cause mortality in healthy men. J Gerontol A Biol Sci Med Sci. 2002;57:B359B365

6. Rantanen T, Harris T, Leveille SG, et al. Muscle strength and body mass index as longterm predictors of mortality in initially healthy men. J Gerontol A Biol Sci Med Sci. 2000;55:M168-M173

7. Katzmarzyk PT, Craig CL. Musculoskeletal fitness and risk of mortality. Med Sci Sports Exerc. 2002;34:740-744.

8. Bassey EJ, Harries UJ. Normal values for handgrip strength in 920 men and women aged over 65 years, and longitudinal changes over 4 years in 620 survivors. Clin Sci (Lond). 1993;84:331-337.

9. Payne N, Gledhill N, Katzmarzyk PT, Jamnik VK, Keir PJ. Canadian musculoskeletal fitness norms. Can J Appl Physiol. 2000;25:430-442.

10. Kell RT, Bell G, Quinney A. Musculoskeletal fitness, health outcomes and quality of life. Sports Med. 1902;31:863-873.

11. Warburton DE, Gledhill N, Quinney A. Musculoskeletal fitness and health. Can J Appl Physiol. 2001;26:217-237.

12. Warburton DE, Glendhill N, Quinney A. The effects of changes in musculoskeletal fitness on health. Can J Appl Physiol. 2001;26:161-216.

13. Guralnik JM, Simonsick EM, Ferrucci L, et al. A short physical performance battery assessing lower extremity function: association with self-reported disability and prediction of mortality and nursing home admission. J Gerontol. 1994;49:M85-M94

14. Hirvensalo M, Rantanen T, Heikkinen E. Mobility difficulties and physical activity as predictors of mortality and loss of independence in the community-living older population. J Am Geriatr Soc. 2000;48:493-498.

15. Balke B, Ware RW. An experimental study of physical fitness in Air Force personnel. US Armed Forces Med J. 1959;10:675-688.

16. Gordon NF, Kohl HW, III, Pollock ML, et al. Cardiovascular safety of maximal strength testing in healthy adults. Am J Cardiol. 1995;76:851-853.

17. Brill PA, Macera CA, Davis DR, Blair SN, Gordon N. Muscular strength and physical function. Med Sci Sports Exerc. 2000;32:412-416.

18. Wilmore JH. Design issues and alternatives in assessing physical fitness among apparently healthy adults in a health examination survey of the general population. In: Drury TF, ed. Assessing Physical Fitness and Physical Activity in Population-based Surveys. Washington, DC: U.S. Government Printing Office; 1989:107-153. 
19. Pollock ML, Bohannon RL, Cooper KH, et al. A comparative analysis of four protocols for maximal treadmill stress testing. Am Heart J. 1976;92:39-46.

20. Pollock ML, Foster C, Schmidt D, et al. Comparative analysis of physiologic responses to three different maximal graded exercise test protocols in healthy women. Am Heart J. 1982;103:363-373.

21. Blair SN, Kampert JB, Kohl HW, et al. Influences of cardiorespiratory fitness and other precursors on cardiovascular disease and all-cause mortality in men and women. JAMA. 1996;276:205-210.

22. Stampfer MJ, Willett WC, Speizer FE, et al. Test of the National Death Index. Am J Epidemiol. 1984;119:837-839.

23. Cox DR. Regression models and life tables (with discussion). J Royal Stat Soc. 1972; Series B 34:187-220.

24. Rantanen T, Era P, Heikkinen E. Physical activity and the changes in maximal isometric strength in men and women from the age of 75 to 80 years. J Am Geriatr Soc. 1997; 45:1439-1445.

25. Schroll M, Avlund K, Davidsen M. Predictors of five-year functional ability in a longitudinal survey of men and women aged 75 to 80. The 1914-population in Glostrup, Denmark. Aging (Milano). 1997;9:143-152.

26. Laukkanen P, Heikkinen E, Kauppinen M. Muscle strength and mobility as predictors of survival in 75-84-year-old people. Age Ageing. 1995;24:468-473.

27. Rantanen T, Guralnik JM, Foley D, et al. Midlife hand grip strength as a predictor of old age disability. JAMA. 1999;281:558-560.

28. Fiatarone MA, Marks EC, Ryan ND, et al. High-intensity strength training in nonagenarians. Effects on skeletal muscle. JAMA. 1990;263:3029-3034.

29. Hurley BF, Roth SM. Strength training in the elderly: effects on risk factors for agerelated diseases. Sports Med. 2000;30:249-268.

30. Pollock ML, Vincent KR. Resistance training for health. The President's Council on Physical Fitness and Sports Research Digest. 1996;Series 2:1-9.

31. Blair SN, Kohl HW, III, Paffenbarger RS, Jr., et al. Physical fitness and all-cause mortality: a prospective study of healthy men and women. JAMA. 1989;262:2395-2401.

\section{Acknowledgments}

This study was supported in part by U.S. Public Health Service research grants from the National Institute on Aging (AG06945) and National Institute of Arthritis and Musculoskeletal and Skin Diseases (AR39715), Bethesda, MD. We thank the physicians and technicians of the Cooper Clinic, Kia Vaandrager and her staff for collecting data for this study, Kenneth H. Cooper, M.D., for initiating the Aerobics Center Longitudinal Study, the MIS and data entry staff of The Cooper Institute, Melba S. Morrow, M.A. for editorial assistance, Neil Gordon, M.D., Ph.D., Principal Investigator, and Patricia Brill, Project Coordinator, of the AR39715 project, for their contributions to that part of the study. 